\title{
Characteristics of stem cells derived from rat fascia: In vitro proliferative and multilineage potential assessment
}

\author{
HING-LOK WONG ${ }^{1,2 *}$, WING-SUM SIU ${ }^{1-3 *}$, CHAK-HEI FUNG ${ }^{1-3}$, CHENG ZHANG $^{1,2}$, \\ WAI-TING SHUM ${ }^{1,2}$, XUE-LIN ZHOU ${ }^{1,2}$, CLARA BIK-SAN LAU ${ }^{1,2}$, JING-FANG ZHANG $^{4,5}$, \\ PING-CHUNG LEUNG ${ }^{1-3,6}$, WEI-MING FU ${ }^{4}$ and CHUN-HAY KO $\mathrm{KO}^{1,2,6}$
}

\author{
${ }^{1}$ Institute of Chinese Medicine; ${ }^{2}$ State Key Laboratory of Phytochemistry and Plant Resources in West China; \\ ${ }^{3}$ Department of Orthopaedics and Traumatology, Chinese University of Hong Kong, Shenzhen, Guangdong 518057; \\ ${ }^{4}$ Institute Guangzhou of Advanced Technology, Chinese Academy of Sciences, Guangzhou, Guangdong 510275; \\ ${ }^{5}$ School of Biomedical Sciences; ${ }^{6}$ CUHK Shenzhen Research Institute, Chinese University of Hong Kong, \\ Shenzhen, Guangdong 518057, P.R. China
}

Received December 2, 2013; Accepted July 1, 2014

DOI: $10.3892 / \mathrm{mmr} .2014 .2967$

\begin{abstract}
Fascia-derived stem cells (FDSCs) were previously isolated from the fascia of the gluteus maximus of the rat. However, the use of FDSCs as a cell source for musculoskeletal tissue engineering has not been compared with that of adipose-derived stem cells (ADSCs) and bone marrow-derived mesenchymal stem cells (BMSCs). Therefore, the present study aimed to compare the mesenchymal stem cell (MSC) and self-renewal stem cell markers, proliferative capacity and multilineage differentiation potential of these stem cells in vitro. The MSC and embryonic stem cell (ESC) marker profiles were compared using flow cytometry and quantitative polymerase chain reaction (qPCR). Their proliferative capacities were compared using 5-bromo-2'-deoxyuridine and MTT assays. Their osteogenic, adipogenic and chondrogenic differentiation potentials were compared using standard staining assays and qPCR. The FDSCs possessed similar cell morphology and immunophenotypic profiles with BMSCs and ADSCs. FDSCs demonstrated a similar expression pattern of ESC markers with ADSCs, which has higher expression of sex determining region Y-box (Sox)2 and octamer-binding
\end{abstract}

Correspondence to: Dr Chun-Hay Ko, CUHK Shenzhen Research Institute, The Chinese University of Hong Kong, 10 2nd Yuexing Road, Nanshan, Shenzhen, Guangdong 518057, P.R. China

E-mail: gohey@cuhk.edu.hk

Dr Wei-Ming Fu, Institute Guangzhou of Advanced Technology, Chinese Academy of Sciences, 1121 Haibin Road, Nansha, Guangzhou, Guangdong 510275, P.R. China

E-mail: wm.fu@giat.ac.cn

*Contributed equally

Key words: fasciology, fascia, stem cells, osteogenesis, adipogenesis, chondrogenesis transcription factor 4, and lower expression of Krüppel-like factor 4, when compared with BMSCs. FDSCs exhibited higher proliferation under serum-deprived conditions $(0.5 \%$ FBS growth medium), and attained higher expression levels of collagen type I, $\alpha 2$ and type II, $\alpha 1$ as well as Sox9 mRNA than ADSCs and BMSCs upon chondrogenic induction. An increased amount of proteoglycan deposition was also observed in the FDSC group. However, lower levels of adipogenic and osteogenic marker expression in FDSCs were detected compared with ADSCs and BMSCs upon adipogenic and osteogenic induction, respectively. FDSCs possessed high chondrogenic potential, low osteogenic and adipogenic differentiation potential and were responsive to the induction signals for collagen-rich fascial structure regeneration. Therefore, FDSCs may represent an improved alternative cell source to conventional ADSCs and BMSCs for musculoskeletal tissue repair and tissue engineering, particularly for collagen-rich structures with poor vasculature.

\section{Introduction}

Mesenchymal stem cells (MSCs) are multipotent adult stem cells, found intrinsically throughout the body following development. They multiply by cell division to replenish dying cells and repair malfunctioning tissues (1). MSCs are capable of differentiating into osteoblasts, adipocytes, chondrocytes, fibroblasts, neuronal tissues, myocytes and tenocytes (2). They are considered to reside in specific areas of each tissue and remain inactive for long periods of time, until they are activated by signals indicating that more cells are required to maintain tissue integrity, or by signals from sites of tissue injury. Different cytokines and growth factors are recruited in stem cell fate regulation, including quiescence, self-renewal, differentiation, apoptosis and mobilization from their original niche (3-5).

MSCs have been found in numerous tissue types of mesenchymal origin, predominantly in bone marrow, but also in adipose tissues, skeletal muscle, connective tissues, teeth and 
visceral organs (6). MSCs from different tissue sources share certain global characteristics but variations do exist among them (7). No specific antigenic phenotypes for MSCs are found, but they share the features of endothelial, epithelial and muscle cells, including CD29, CD44, CD90 and CD105 (8). MSCs do not express the typical hematopoietic antigens, such as CD45 and CD34. It has been reported that there were differences in yield, expansion and multipotent differentiation potential among MSCs isolated from bone marrow, synovium, periosteum, adipose tissue and muscle $(9,10)$. MSCs isolated from alveolar bone demonstrated less chondrogenic and adipogenic potential than those isolated from iliac bone (11). Improved understanding of the characteristics of stem cells from different sources may facilitate the identification of an improved cellular source for tissue engineering. For example, Rui et al (12) found that tendon-derived stem cells (TDSCs) possess higher BMP2 receptor expression to facilitate osteogenic differentiation when compared with bone marrow-derived MSCs (BMSCs), and therefore, implicating TDSCs to be an attractive source for tendon-bone junction healing. Numerous comparisons have been made for adipose tissue-derived stem cells (ADSCs) and BMSCs, illustrating that ADSCs were viable alternatives, even as a more preferable source for cell therapy or pre-clinical drug testing than BMSCs (13-15).

While the tendons attach muscle to bones and ligaments connect bone to bone, forming and maintaining joints, the fascia is a collective tissue that essentially holds the entire body together. The fascia is also defined as a 'web of tissue' that surrounds every muscle, bone and organ in the body and holds everything in place. It is essential for the body's self-healing process, as once the epidermis is penetrated, it is the fascia that staves off infection and further damage to the interior of the body. Similar to all other tissues in the human body, the fascia becomes inflamed when damaged, causing discomfort and pain. However, similar to anterior cruciate ligament injuries, a torn fascia (i.e. plantar fascia injury) is always associated with a slow and poor recovery. There is high prospective to apply tissue engineering strategies to improve the fascia healing process by using the stem cells within. Previously, Tao et al (16) suggested a novel term called 'fasciology', hypothesizing that the fascial network distributed throughout the body constructs a supporting-storing system to nurture surrounding internal organs. The essence of Traditional Chinese Medicine meridians and acupuncture may be explained in the view of fascial anatomy. Over the last decade, this novel theory has been conceptually verified through finding evidence from the mechanism of acupuncture and Traditional Chinese Medicine, evolutionary biology, holistic therapies and complementary medicine (17). Li et al (18) also discovered that cells isolated from the fascia of the gluteus maximus possessed chondrogenic potential, which was different from neighboring muscle-derived stem cells. However, despite these findings, to the best of our knowledge, there is no comprehensive report to date that has characterized the other stem cell properties of those cells isolated from rat fascia structures.

Based on the aforementioned studies reporting that the fascia is an intact structure that functions to connect muscles and organs, it was hypothesized that resident fascia-derived stem cells (FDSCs) should possess high chondrogenic, low osteogenic and adipogenic differentiation potential and responsiveness to the induction signals for collagen-rich fascial structure regeneration. Therefore, FDSCs may represent an improved alternative cell source compared with conventional ADSCs and BMSCs for musculoskeletal tissue repair and tissue engineering. The present study aimed to compare the stem cell marker expression, immunophenotypic profile, proliferative capacity and multilineage differentiation potential of rat FDSCs, ADSCs and BMSCs in vitro.

\section{Materials and methods}

Isolation and culture of rat FDSCs, ADSCs and BMSCs. The Animal Research Ethics Committee of the Chinese University of Hong Kong (Hong Kong, China) approved all of the experiments. Eight male Sprague-Dawley rats (10 weeks old) weighing $250 \mathrm{~g}$ were used in the present study. FDSCs, ADSCs and BMSCs were isolated from the same animals. The procedures for the isolation of FDSCs, ADSCs and BMSCs are described as follows. FDSCs were isolated from the fascia of the left gluteus maximus of the rats, which were carefully detached from the muscle using surgical scissors. ADSCs were isolated from the inguinal fat pad. Both FDSCs and ADSCs were enzymatically isolated from their extracellular matrix using type I collagenase ( $3 \mathrm{mg} / \mathrm{ml}$; Sigma-Aldrich, St. Louis, MO, USA) and passed through a 70-mm cell strainer (Becton-Dickinson, Franklin Lakes, NJ, USA) to yield single-cell suspensions. The BMSCs were isolated from the bone of femora by centrifugation as described previously (19). Isolated FDSCs, ADSCs and BMSCs were cultured in a growth medium $[\alpha-\mathrm{MEM}$ (Invitrogen Life Technologies, Carlsbad, CA, USA) containing 10\% fetal bovine serum and $1 \%$ penicillin/streptomycin (Invitrogen Life Technologies)] and seeded at a density of $2 \times 10^{5} / \mathrm{cm}^{2}$ at $37^{\circ} \mathrm{C}$ in $95 \%$ humidified air and $5 \% \mathrm{CO}_{2}$. On day 7 , all non-adherent cells were removed followed by a medium change twice a week. The monolayer of adherent cells was trypsinized by $0.25 \%$ trypsin-EDTA when it reached half-confluence and reseeded at a density of $1 \times 10^{4} / \mathrm{cm}^{2}$ [passage 1 (P1)]. Passage 2 (P2) culture was used for all characterization and in vitro assays.

Colony formation unit assay, cell proliferation and viability assay. The colony-forming unit (CFU) assay is used to quantify functional stem cells. Briefly, 500 FDSCs, ADSCs and BMSCs at P2 were seeded in 100-mm sterile petri dishes and cultured for 14 days. The colonies formed were stained with $1 \%$ crystal violet (Sigma-Aldrich) in methanol for $30 \mathrm{~min}$. For the cell proliferation assay, FDSCs, ADSCs and BMSCs at P2 were seeded in a 96-well plate at a density of 5,000 cells/well and incubated at $37^{\circ} \mathrm{C}, 5 \% \mathrm{CO}_{2}$. At day 2 , cell proliferation was assessed using a 5-bromo-2-deoxyuridine (BrdU) assay kit (Roche Applied Science, Penzberg, Germany) according to the manufacturer's instructions. The absorbance was measured at an optical density (OD) of $450 \mathrm{~nm}$, using a $\mu$ Quant $^{\mathrm{TM}}$ Microplate Spectrophotometer (BioTek Instruments, Inc., Winooski, VT, USA). The relative cell viability in metabolically active cells was also determined by measuring the reduction of MTT dye (Sigma, St. Louis, MO, USA) to blue formazan crystals at an OD of $540 \mathrm{~nm}$, following a $3 \mathrm{~h}$ incubation at $37^{\circ} \mathrm{C}$.

Immunophenotypic profile. The immunophenotypic identities of the FDSCs, ADSCs and BMSCs were characterized by flow 
Table I. Primer sequences for self-renewal stem cell markers and differentiation markers.

\begin{tabular}{|c|c|c|c|}
\hline Symbol & Description & Primer sequence $\left(5^{\prime} \rightarrow 3^{\prime}\right)$ & Accession No. \\
\hline Oct4 & POU class 5 homeobox 1 & $\begin{array}{l}\text { (F) GTCCCTAGGTGAGTCGTCCT } \\
\text { (R) TGGAAGCTTAGCCAGGTTCG }\end{array}$ & NM_001009178 \\
\hline Sox 2 & SRY-box 2 & $\begin{array}{l}\text { (F) GAGGAGGAGAGCGACTGTTT } \\
\text { (R) CTGGCGGAGAATAGTTGGGG }\end{array}$ & NM_001109181 \\
\hline Klf4 & Kruppel-like factor 4 & $\begin{array}{l}\text { (F) GCCACCCACACTTGTGACTA } \\
\text { (R) TTCTCGCCTGTGTGAGTTCG }\end{array}$ & NM_053713 \\
\hline Runx2 & $\begin{array}{l}\text { Runt-related } \\
\text { transcription factor } 2\end{array}$ & $\begin{array}{l}\text { (F) CACAAGTGCGGTGCAAACTT } \\
\text { (R) GCAGCCTTAAATATTACTGCATGG }\end{array}$ & NM_053470 \\
\hline Alpl & Alkaline phosphatase & $\begin{array}{l}\text { (F) GATGGTATGGGCGTCTCCAC } \\
\text { (R) TCTTGGAGAGAGCCACAAAGG }\end{array}$ & NM_013059 \\
\hline OPN & Osteopontin & $\begin{array}{l}\text { (F) CCGAGGTGATAGCTTGGCTT } \\
\text { (R) CTCTTCATGCGGGAGGTGAG }\end{array}$ & NM_012881 \\
\hline ON & Osteonectin & $\begin{array}{l}\text { (F) ACCTGGACTACATCGGACCA } \\
\text { (R) ACCAGGACGTTTTTGAGCCA }\end{array}$ & NM_012656 \\
\hline $\mathrm{C} / \mathrm{EBP} \alpha$ & $\begin{array}{l}\text { CCAAT/enhancer binding } \\
\text { protein } \alpha\end{array}$ & $\begin{array}{l}\text { (F) GGCCATTCGCGACCC } \\
\text { (R) ACTCCATGGGGGAGTTAGAGT }\end{array}$ & NM_012524 \\
\hline $\operatorname{PPAR} \gamma$ & $\begin{array}{l}\text { Peroxisome proliferator- } \\
\text { activated receptors } \gamma\end{array}$ & $\begin{array}{l}\text { (F) CCTGTTGACCCAGAGCATGG } \\
\text { (R) GGTCCACAGAGCTGATTCCG }\end{array}$ & NM_013124 \\
\hline AP2 & $\begin{array}{l}\text { Adipocyte fatty } \\
\text { acid-binding protein }\end{array}$ & $\begin{array}{l}\text { (F) TCGTCATCCGGTCAGAGAGT } \\
\text { (R) CCAGCTTGTCACCATCTCGT }\end{array}$ & U75581.1 \\
\hline Adipsin & Complement factor D & $\begin{array}{l}\text { (F) TGGGGCAATCACCAAGAACA } \\
\text { (R) CGAGATCCCCACGTAACCAC }\end{array}$ & NM_001077642 \\
\hline Sox9 & SRY-box containing gene 9 & $\begin{array}{l}\text { (F) TGGGAGCGACAACTTTACCA } \\
\text { (R) GAGGAGGAGGGAGGGAAAAC }\end{array}$ & XM_001081628 \\
\hline Col1a2 & Collagen, type I, $\alpha 2$ & $\begin{array}{l}\text { (F) GAGGCTTCTACAGGGCTGAC } \\
\text { (R) CTTAAGTCACGGCATGTGCG }\end{array}$ & NM_053356 \\
\hline Col2a1 & Collagen, type II, $\alpha 1$ & $\begin{array}{l}\text { (F) GTTCACGTACACTGCCCTGA } \\
\text { (R) AAGGCGTGAGGTCTTCTGTG }\end{array}$ & NM_012929 \\
\hline Agg & Aggrecan & $\begin{array}{l}\text { (F) GAAGTGGCGTCCAAACCAAC } \\
\text { (R) AGCTGGTAATTGCAGGGGAC }\end{array}$ & NM_022190.1 \\
\hline
\end{tabular}

F, forward; R, reverse; SRY, sex-determining region $\mathrm{Y}$.

cytometry using the CANTO 11 flow cytometer with the FACs Diva software (BD Biosciences, San Diego, CA, USA). All of the antibodies were obtained from AbD Serotec (Raleigh, NC, USA) and used at 1:100 dilutions. The antibodies used were against cell surface antigens, CD44 (cat. no.: MCA643F), CD71 (cat. no.: MCA155PE), CD90 (cat. no.: MCA47PE), CD106 (positive; cat. no.: MCA4633F), and hematopoietic markers CD11b (cat. no.: MCA275FT) and CD45 (negative; cat. no.: MCA43FT).

Stem cell marker analysis. The expression of pluripotency and self-renewal stem cell markers octamer-binding transcription factor 4 (Oct4), sex determining region Y (SRY)-box (Sox)2 and Krüppel-like factor 4 (Klf4) in FDSCs, ADSCs and BMSCs at P2 were compared using quantitative polymerase chain reaction (qPCR). The amount of mRNA was determined using the Quanti-Fast SYBR Green RT-PCR kit (Qiagen, Hilden, Germany) with a validated primer set specific for the target genes from Qiagen (as listed in Table I) in the CFX96 Real-Time PCR Detection system (Bio-Rad, Hercules, CA, USA). The relative expression of the qPCR product was calculated using the comparative $2^{-\Delta \Delta \mathrm{Ct}}$ method. The endogenous control glyceraldehyde-3-phosphate dehydrogenase (GAPDH) mRNA was used for normalization.

Assessment of differentiation potential. For the differentiation studies, FDSCs, ADSCs and BMSCs at P2 were seeded in six-well plates at a density of $3 \times 10^{5}$ cells/well. Following three days, the growth medium was replaced with osteogenic medium (growth medium supplemented with $100 \mathrm{nM}$ dexamethasone, $50 \mu \mathrm{g} / \mathrm{ml}$ ascorbate-2-phosphate and $10 \mathrm{mM}$ $\beta$-glycerol phosphate), or adipogenic medium (growth 


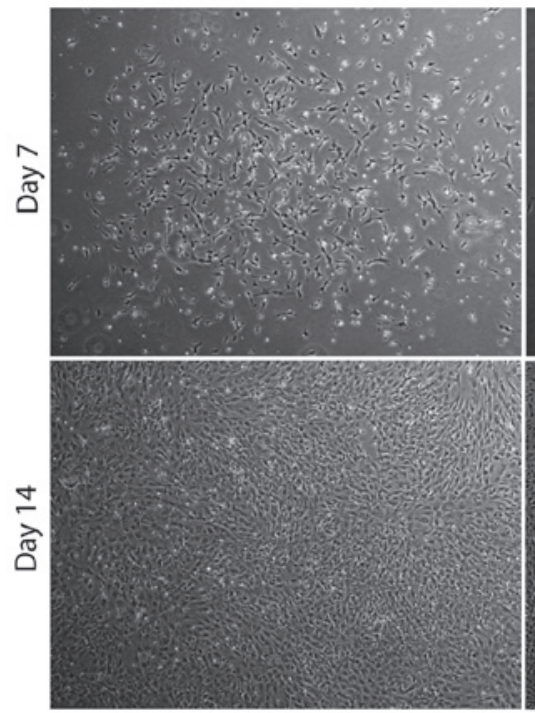

BMSCs

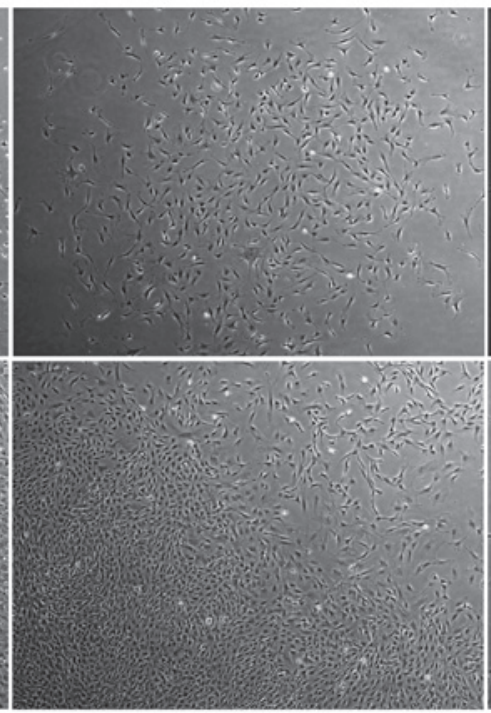

ADSCs

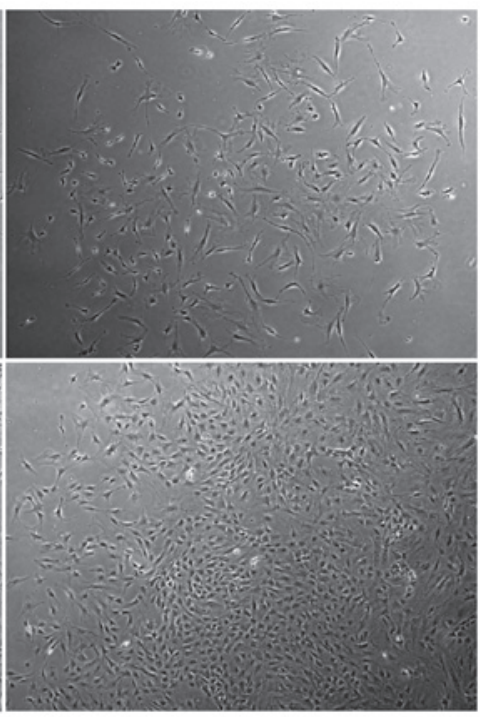

FDSCs

Figure 1. Cell morphology of isolated stem cells. The cells were viewed under an inverted light microscope (magnification, x100). All stem cell populations demonstrated a fibroblast-like shape in vitro. Upper panels, cells grown for 7 days; lower panels, cells growth for 14 days. FDSCs, fascia-derived stem cells; ADSCs, adipose-derived stem cells; BMSCs, bone marrow-derived mesenchymal stem cells.

medium supplemented with $1 \mu \mathrm{M}$ dexamethasone, $50 \mu \mathrm{g} / \mathrm{ml}$ insulin, $0.5 \mathrm{mM}$ methyl-isobutylxanthine and $100 \mu \mathrm{M}$ indomethacin) with the medium changed twice a week for 14 days. The chondrogenetic potential of FDSCs was induced by the StemPro ${ }^{\circledR}$ Chondrogenesis Differentiation kit (Invitrogen Life Technologies), according to the manufacturer's instructions. Briefly, $1.6 \times 10^{7}$ cells were used to generate a micromass culture for 28 days. The differentiated cells were visualized using alizarin red $\mathrm{S}$, oil red $\mathrm{O}$ and alcian blue staining for successful osteogenesis, adipogenesis and chondrogenesis, respectively. To compare the osteogenic, adipogenic and chondrogenic potential of the FDSCs with ADSCs and BMSCs, the mRNA expression of the marker genes was measured at day 7 (for ostegenesis and adipogenesis) and day 14 (for chondrogenesis). The amount of mRNA was determined using the Quanti-Fast SYBR Green RT-PCR kit (Qiagen) with a validated primer set specific for the target genes from Qiagen (as listed in Table I) in the CFX96 Real-Time PCR Detection System (Bio-Rad, United States). The relative expression of the qPCR product was calculated using the comparative $2^{-\Delta \Delta \mathrm{Ct}}$ method. The endogenous control GAPDH mRNA was used for normalization.

Statistical analysis. The differences between groups were tested by one-way analysis of variance, followed by a post-hoc Dunn's test. All statistical analyses were performed with SPSS 15.0 (SPSS, Inc., Chicago, IL, USA). P<0.05 was considered to indicate a statistically significant difference between values. Values are expressed as the mean \pm standard derivation.

\section{Results}

Cell morphology. FDSCs from the fascia of the left gluteus maximus of the rats were isolated $(n=3)$ and the cells were well attached on culture vessels until confluent. Similar fibroblast-like cell morphology was observed when compared
Table II. Immunophenotypic profile of three stem cell populations. Surface markers are indicated as positive (+) and negative (-).

\begin{tabular}{lccc}
\hline Surface markers & BMSCs & ADSCs & FDSCs \\
\hline CD11b & - & - & - \\
CD31 & - & - & - \\
CD34 & - & - & - \\
CD44 & + & + & + \\
CD45 & - & - & - \\
CD71 & + & + & + \\
CD90 & + & + & + \\
CD106 & + & - & - \\
\hline
\end{tabular}

FDSCs demonstrated the same immunophenotypic profile as ADSCs which was similar to that of BMSCs, except for a negative expression in CD106. FDSCs, fascia-derived stem cells; ADSCs, adipose-derived stem cells; BMSCs, bone marrow-derived mesenchymal stem cells.

with those for BMSCs and ADSCs under similar growth conditions (Fig. 1). The cells were reseeded and expanded to a number of passages $(n>5)$ without slowing proliferation, which proceeded with a doubling time of $\sim 14-21$ days.

Comparison of cell proliferation. The CFU assay identified that the size of the colonies from FDSCs was generally bigger than that of BMSCs and similar to that of ADSCs (Fig. 2A). An increased number of colonies were observed for the FDSCs compared with the other two cell types. In addition, FDSCs exhibited significantly higher proliferation potential than BMSCs under different serum concentrations, as indicated by the BrdU and MTT assays (Fig. 2B and C). However, in the $10 \%$ FBS medium, both ADSCs and FDSCs exhibited similar proliferation patterns. In the lower serum-containing growth medium 


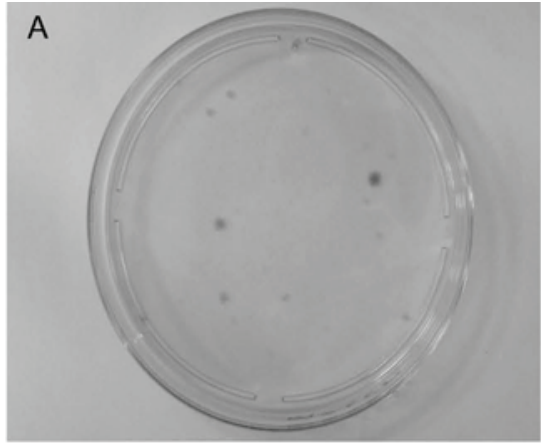

BMSCS

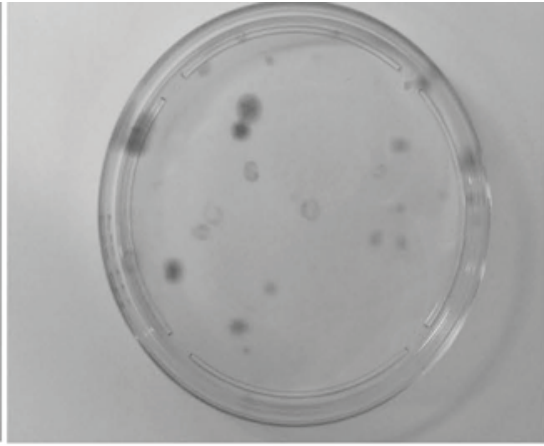

ADSCS

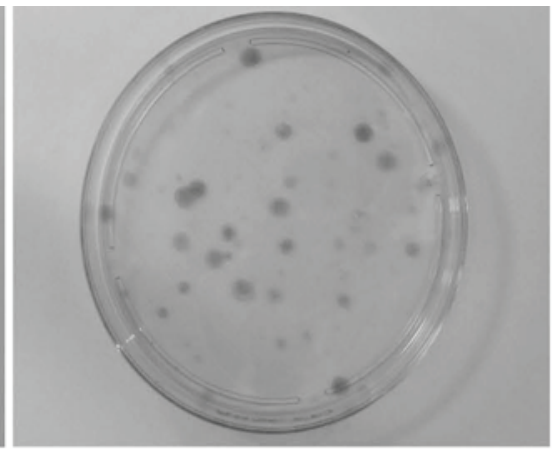

FDSCS
$0.5 \%$ FBS

B

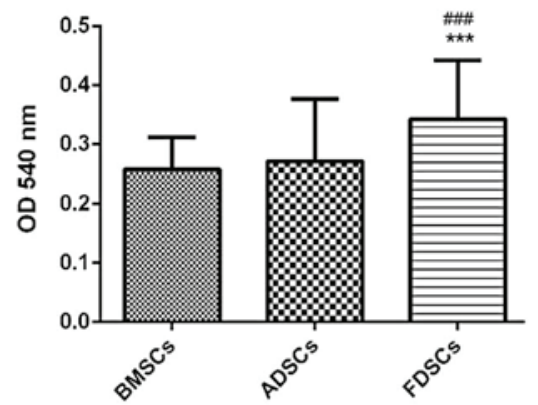

C

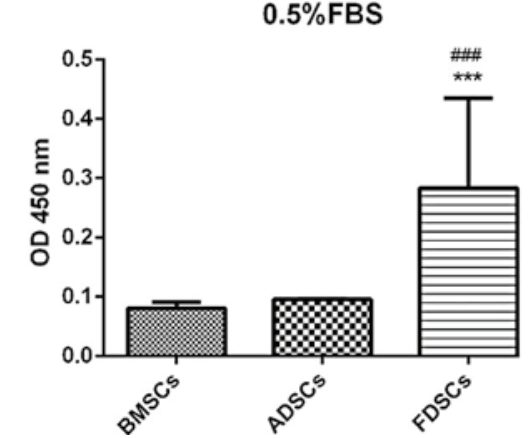

$10 \%$ FBS

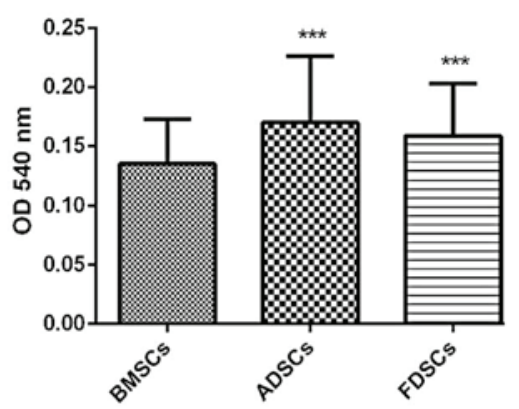

$10 \%$ FBS

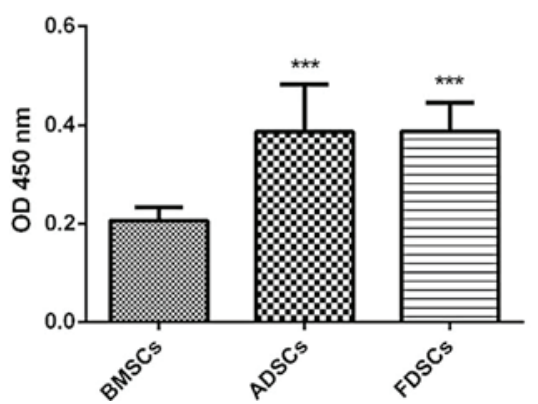

Figure 2. Cell proliferation assays. (A) Colony-forming unit assay. Size and number of stained colonies from the individual populations were compared. At the same seeding cell number, the FDSCs formed colonies of the highest number and biggest in size among the three populations. (B) MTT assay. OD at $540 \mathrm{~nm}$ was measured. FDSCs attained the highest degree of proliferation among the populations under serum-deprived conditions (0.5\% FBS). Under normal serum concentration (10\% FBS), ADSCs exhibited significantly higher proliferation potential than BMSCs and FDSCs. (C) 5-bromo-2'-deoxyuridine assay. OD at $450 \mathrm{~nm}$ was measured. Under normal serum conditions, both ADSCs and FDSCs exhibited similar proliferation patterns. While under lower serum-deprived

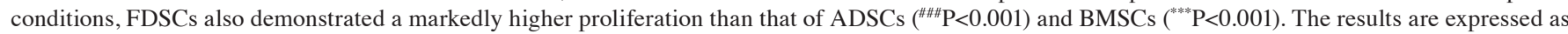
the mean values \pm standard deviation of data from experiments performed in triplicate. FDSCs, fascia-derived stem cells; ADSCs, adipose-derived stem cells; BMSCs, bone marrow-derived mesenchymal stem cells; OD, optical density; FBS, fetal bovine serum.

$(0.5 \%)$, FDSCs attained a markedly higher proliferation than that of ADSCs in both BrdU and MTT assays (both $\mathrm{P}<0.05$ ).

Immunophenotypic profile. Flow cytometry using antibodies against specific surface antigens of stem cells was performed. The immunophenotypic profile of BMSCs was CD11b ${ }^{-}$CD31', CD34-, CD44 ${ }^{+}, \mathrm{CD}^{-} 5^{-}, \mathrm{CD}^{+}{ }^{+}, \mathrm{CD}^{+}{ }^{+}$and $\mathrm{CD} 106^{+}$(Table II). FDSCs demonstrated an identical immunophenotypic profile to that of ADSCs, which was similar to that of BMSCs except for no of expression of CD106.

Comparison of stem cell marker expression. Stem cell marker expression was assessed by qPCR. Both FDSCs and ADSCs exhibited significantly higher expression of Sox 2 and Oct4 than BMSCs by 5- to 13 -fold ( $\mathrm{P}<0.001$; Fig. 3$)$. The expression of Sox 2 in FDSCs was evidently lower than that in ADSCs $(\mathrm{P}<0.001)$. However, a lower expression of Klf4 in ADSCs $(\mathrm{P}<0.05)$ and FDSCs as compared with that in BMSCs was observed.

Comparison of differentiation potential. All three cell populations demonstrated the ability of in vitro differentiation (Fig. 4). MSCs exhibited the highest osteogenic potential, as demonstrated by marked staining with alizarin red S. All of the cell populations demonstrated an adipogenic potential with a higher number of adipocytes formed in the ADSCs group, demonstrated by oil red $\mathrm{O}$ staining. Chondrogenic potential (proteoglycan deposition) was demonstrated by alcian blue staining and FDSCs demonstrated improved staining among the three cell populations. 
Sox2

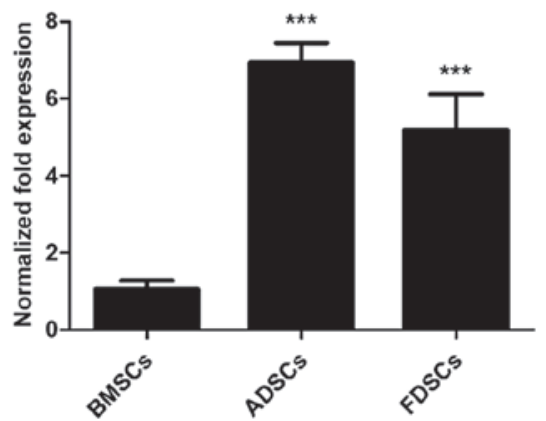

KIf4

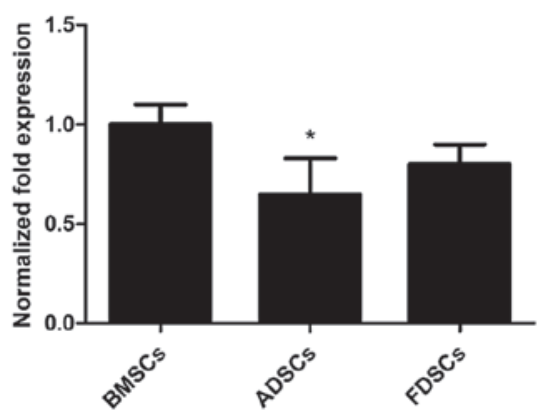

Oct4

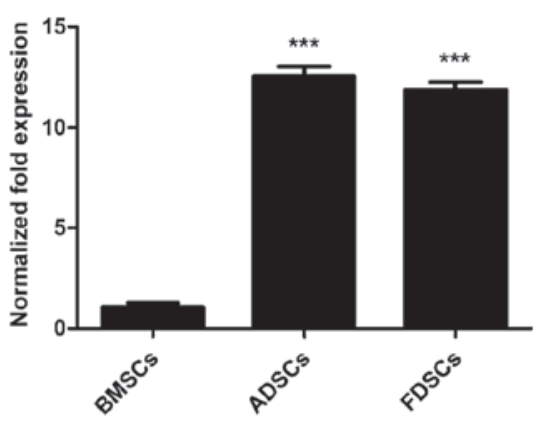

Figure 3. Expression of stem cell markers in the cell populations was studied by quantitative polymerase chain reaction. The values were calculated with the reference to GAPDH and the samples were run in triplicate. FDSCs and ADSCs expressed higher levels of Sox 2 and Oct4 (**** P $<0.001)$ and lower levels of klf4 than BMSCs ( $\mathrm{P}<0.05$ in ADSCs). The results are expressed as the mean values \pm standard deviation of data from experiments performed in triplicate. FDSCs, fascia-derived stem cells; ADSCs, adipose-derived stem cells; BMSCs, bone marrow-derived mesenchymal stem cells; Oct4, octamer-binding transcription factor 4; Sox2, sex-determining region Y-box 2; klf4, Krüppel-like factor 4.

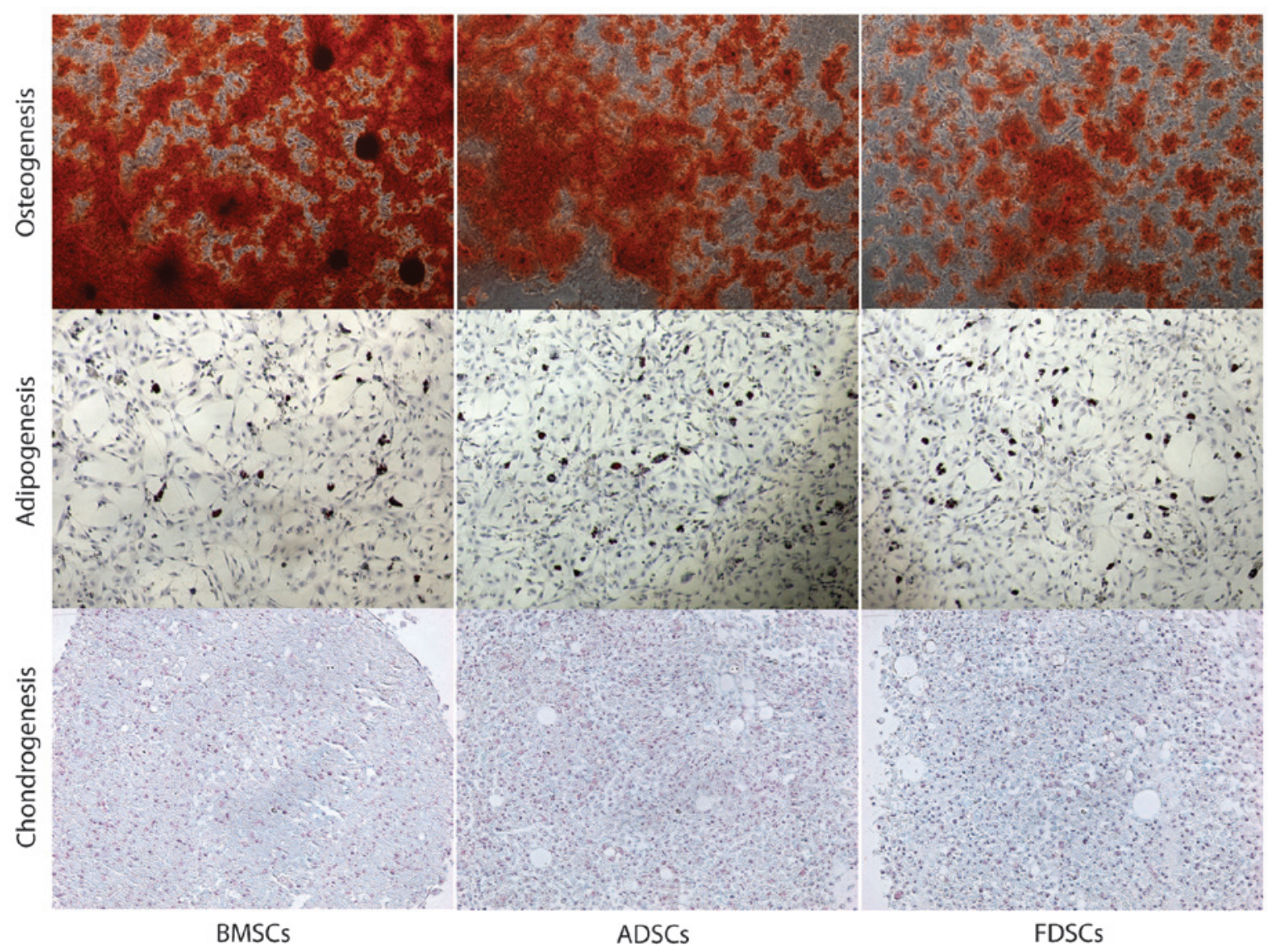

Figure 4. Osteogeinc, adipogenic and chondrogenic in vitro differentiation. Upon differentiation, the cells were then stained with alizarin red S for osteogenic differentiation, oil red $\mathrm{O}$ for adipogenic differentiation and alcian blue for chondrogenic differentiation. Marked staining of alizarin red $\mathrm{S}$ was observed in BMSCs. An increased number of oil red O-positive cells were observed in ADSCs and increased alcian blue staining (proteoglycan deposition) was observed in the FDSC population (magnification, $\mathrm{x} 40$ ). The experiments were performed in triplicate. FDSCs, fascia-derived stem cells; ADSCs, adipose-derived stem cells; BMSCs, bone marrow-derived mesenchymal stem cells.

Comparison of gene expression. The expression levels of osteogenic markers runt-related transcription factor 2 (Runx2), alkaline phosphatase (ALP), osteopontin (OPN) and osteonectin $(\mathrm{ON})$ in both ADSCs and FDSCs (all $\mathrm{P}<0.001)$ were found to be notably lower than those in the BMSCs (Fig. 5A). The expression pattern of these genes in FDSCs was similar to that of ADSCs. Among the three cell populations,
ADSCs exhibited the significantly highest expression of the adipogenic markers CCAAT/enhancer binding protein $\alpha(\mathrm{C} / \mathrm{EBP} \alpha)$, peroxisome proliferator-activated receptor $\gamma$ (PPAR $\gamma$ ), AP2 and adipsin, particularly on AP2 expression which was 25 -fold higher (all $\mathrm{P}<0.001$; Fig. 5B). Meanwhile, increased expression of adipocyte fatty acid binding protein (AP2) and adipsin only were observed in FDSCs $(\mathrm{P}<0.001)$. 
A

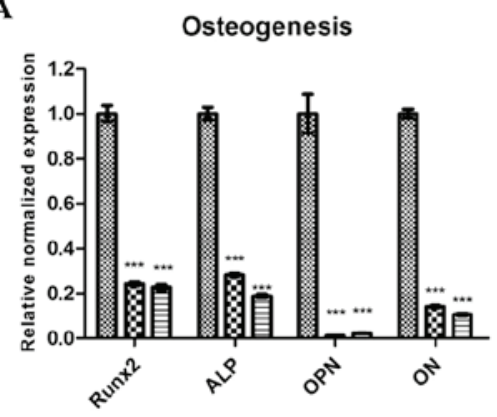

B

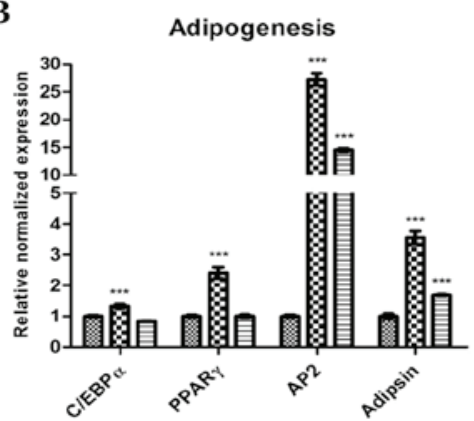

C

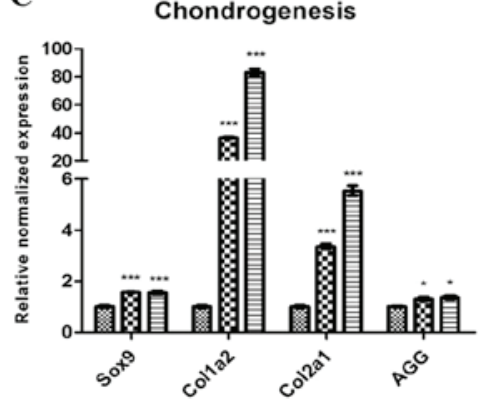

Figure 5. mRNA expression of osteogenic, adipogenic and chondrogenic markers was analyzed by quantitative polymerase chain reaction. (A) Expression of all the osteogenic markers Runx2, ALP, OPN and ON were significantly decreased in ADSCs and FDSCs ( $\left.{ }^{* * *} \mathrm{P}<0.001\right)$. (B) The highest expression of all adipogenic markers, C/EBP $\alpha$, PPAR $\gamma$, AP2 and Adipsin, was found in ADSCs $\left({ }^{* * *} \mathrm{P}<0.001\right)$, particularly for AP2 expression ( $>25$-fold increase). In FDSCs, significantly higher expression of AP2 and Adipsin only as compared with that in BMSCs were observed $\left({ }^{* * *} \mathrm{P}<0.001\right)$. (C) Increased expression of the chondrogenic markers Sox9, Col1a2, Col2a1 ( $\left.{ }^{* * *} \mathrm{P}<0.001\right)$, and AGG $\left({ }^{*} \mathrm{P}<0.05\right)$ were observed in both ADSCs and FDSCs, while the expression of Colla2 and Col2a1 was significantly increased in FDSCs. The values were calculated with reference to GAPDH and the results are expressed as the mean values \pm standard deviation of data from experiments in triplicate. FDSCs, fascia-derived stem cells; ADSCs, adipose-derived stem cells; BMSCs, bone marrow-derived mesenchymal stem cells; Runx2, runt-related transcription factor 2; ALP, alkaline phosphatase; OPN, osteopontin; ON, osteonectin; C/EBP $\alpha$, CCAAT/enhancer binding protein $\alpha$; PPAR $\gamma$, peroxisome proliferator-activated receptor $\gamma$; AP2, adipocyte fatty acid binding protein; Sox2, sex-determining region Y-box 2; Colla2, collagen, type I, $\alpha$ 2; Col2a1, collagen, type II, $\alpha$ 1; AGG, aggrecan.

Markedly higher expression ( 80-fold to that in the BMSCs) of chondrogenic markers collagen, type I, $\alpha 2$ (Col1a2), together with collagen, type II, $\alpha 1$ (Col2a1; >5-fold), were also observed in the FDSCs $(\mathrm{P}<0.001$; Fig. 5C). High expression of these genes was observed in ADSCs, but their expression was approximately half of that of the FDSCs $(\mathrm{P}<0.001)$. The expression of the other chondrogenic markers Sox $9(\mathrm{P}<0.001)$ and aggrecan $(\mathrm{AGG})(\mathrm{P}<0.05)$ was similar in the ADSCs and FDSCs and their expression was marginally higher than that in the BMSCs.

\section{Discussion}

The fascia first received attention as an important structure in the $1930 \mathrm{~s}$, but few studies were performed investigating its significance for several decades (20). With technological advancements, however, including imaging and anatomical technologies, the fascia structure has been attracting increasing attention. A growing number of studies and evidence have demonstrated that multipotent stem cells should be resided in the fascia (21). Skeletal muscle perimysium, a sheath of connective tissue that segregates skeletal muscle fascicles and fibers, has a similar histology, structure and function to fascia, but is different in the scale of muscle structure. It was further hypothesized that non-myogenic cells within skeletal muscle, likely associated with endomysium and perimysium, may possess chondrogenic potential. However, there is no known physical method to isolate these tissues from skeletal muscle, considering its super-structural complexity. Therefore, the presence of chondrogenic cells in the skeletal muscle of a Fischer 344 rat gluteus maximus muscle was also investigated by isolating a heterogeneous population of muscle-derived cells, which were then examined for the presence of cells with chondrogenic potential (18). The present study successfully isolated cells from the superficial fascia of the limbs of adult rats. To the best of our knowledge, the present study was the first to isolate and characterize rat FDSCs in vitro. These fascia-derived cells had universal MSC characteristics, including clonogenicity, high proliferative potential at reduced serum conditions, MSC marker expression and multidifferentiation potential, including osteogenesis, adipogenesis and chondrogenesis.

The present study compared the immunophenotypic profiles of FDSCs, ADSCs and BMSCs, and found a similar expression pattern of CD44, CD71 and CD90, as well as a difference in the expression of CD106. The BMSCs expressed CD106, which was not detected in ADSCs and FDSCs. The presence of CD106 is controversial in ADSCs. Schäffler and Büchler (22) defined the surface marker set for ADSCs, which included CD106. However, De Ugarte et al (23) and Zuk et al (24) found that CD106 was absent in ADSCs. The present study also demonstrated that there was minimal contamination with hematopoietic and endothelial cells in the culturing system, as evidenced by the weak expression of CD34, CD45 and CD31 in the cultured cells. FDSC propagation (P2) demonstrated the high purity of the cells exhibiting markers similar to BMSCs and ADSCs and negativity for hematopoietic markers suggested that they possibly attained stem cell phenotype characteristics.

The proliferation capacity of stem cells is important with regard to their application in cell therapy. A number of previous studies have indicated that stem cells from different sources exhibited differences in proliferation and differentiation potential, implying that selecting the appropriate cell source for musculoskeletal tissue engineering is significant (25). In the present study, the proliferation capacity of FDSCs was compared with that of ADSCs and BMSCs in media containing 0.5 and $10 \%$ of FBS, using the BrdU and MTT assays. In medium containing $10 \% \mathrm{FBS}$, it was identified that the FDSCs had a similar proliferative response to that of the ADSCs, but with a higher proliferation capacity than the BMSCs. This observation was consistent with previous studies demonstrating that ADSCs exhibited a higher proliferation capacity than BMSCs $(26,27)$. Proliferation studies were also performed, using $0.5 \%$ FBS medium. Of note, it was identified that FDSCs exhibited a higher proliferation than both the 
BMSCs and ADSCs. This may imply that the nutrient supply in the microenvironment may alter the stem cells' proliferation capacity. Potier et al (28) reported that serum starvation and deprivation of growth factors may promote premature aging in MSCs and studies of MSCs in a hypoxic environment, indicating that serum starvation may be associated with marked cell death. By contrast, the present study substantiated that FDSCs function effectively under conditions of serum deprivation. Further investigation is required to determine whether FDSCs exhibit and maintain a hypoxic environment within the appropriate peripheral musculoskeletal tissues with poor vasculature in vivo, to determine its possible clinical applications.

The FDSCs isolated in the present study expressed a number of key embryonic self-renewal stem cell marker genes, including Oct4, Sox 2 and Klf-4. It has been demonstrated that Oct4 transcription factors are critical for stem cell fate selection, in addition to their roles in maintaining the pluripotency and self-renewal capacity in mesenchymal stem cells (29). Oct4 has often been used as a marker of stemness, as differentiated cells demonstrated reduced expression of this marker. Several studies have suggested that Oct4 is essential in sustaining self-renewal capacity of adult somatic stem cells $(30,31)$. In addition, Oct4 binds to DNA cooperatively with Sox 2 at non-palindromic sequences to activate transcription of key pluripotency factors. It has been demonstrated that differentiation signals modulate the expression of Oct 4 and Sox2, such that the induction of Oct4 suppressed neural ectodermal differentiation and promoted mesendodermal differentiation, whereas induction of Sox 2 inhibited mesendodermal differentiation and promoted neural ectodermal differentiation (32). Klf-4 DNA-binding protein has been recently found to be important in regulating MSC transcriptional activity and controlling cell fate (33). In the present study, the FDSCs and ADSCs demonstrated high levels of expression of Oct4 and Sox 2 as compared with that in BMSCs. The higher expression of Oct4 in FDSCs as compared with that in BMSCs and the comparatively lower expression of Sox 2 in FDSCs than that in ADSCs, as observed in the present study, may favor mesendodermal lineage choice of FDSCs when compared with both cell types.

In the present study, it was confirmed that FDSCs, ADSCs and BMSCs have the potential to differentiate into osteogenic, adipogenic and chondrogenic lineages. However, BMSCs have greater osteogenesis potential, while ADSC have a greater adipogenic potential and FDSCs have a greater chondrogenic potential, as evidenced by the increment of expression of their corresponding differentiation markers when compared with those of the other two stem cell populations. In previous years, stem cells have generated increasing interest considering their potential therapeutic use. Previous studies have provided clear evidence that multipotent adult stem cells exist in numerous organs and tissues, including bone marrow, muscle, fat, periosteum and synovial membrane from both rodents and humans $(9,10,15)$. A number of studies have suggested that different stem cells may share common properties for single targeted stem cell therapy (34). For instance, previous studies have suggested that equal or comparable osteogenic capacity were found between ADSCs and BMSCs $(24,35)$. Therefore, ADSCs are attractive for musculoskeletal tissue engineering, since adipose tissue possesses abundant and easily accessible MSCs. However, the present study, in parallel with certain recent data $(36,37)$, suggested that the differentiation potential of the stem cells from different origin may not be identical. Stem cells from different sources may represent distinct cell populations that are at different lineage-specific commitment with distinct biological properties (38). Such differences in differentiation potential may be due to the inherent differences between FDSCs, ADSCs and BMSCs. Therefore, selecting an improved stem cell source for therapeutic use and tissue engineering is required.

In conclusion, a population of stem cells was isolated from the fascia tissue of rats, which exhibited universal stem cell characteristics, including clonogenicity, proliferative capacity, multipotent potential and MSC and ESC marker expression. In addition, these FDSCs were more chondrogenic when compared with ADSCs and BMSCs. The feasibility of isolating stem cells from rat fascia tissues may provide new opportunities for investigating FDSCs for tissue engineering and improving the understanding of the role and mobility of FDSCs in musculoskeletal tissue healing. Additional comparative and functional in vitro and in vivo studies are required to verify these findings to finally provide a better understanding of the biological differences of MSCs from different sources and to identify the most suitable MSCs for treatment of specific diseases. Furthermore, the discovery of FDSCs provided a possible functional role of the fascia structure, namely that of an active support-storage system to their surrounding internal organs.

\section{Acknowledgements}

This study was financially supported by the National Natural Science Foundation of China (grant no. 81200651). This research project was also supported in part by the grants of the State Key Laboratory of Phytochemistry and Plant Resources in West China (CUHK) from HKSAR and CUHK, the Focused Innovations Scheme (Major Area Scheme A - Phase 2) of the Chinese University of Hong Kong and internal funding from the Institute Guangzhou of Advanced Technology, Chinese Academy of Science, Guangzhou.

\section{References}

1. Mundra V, Gerling IC and Mahato RI: Mesenchymal stem cell-based therapy. Mol Pharm 10: 77-89, 2013.

2. Gimble JM, Guilak F, Nuttall ME, et al: In vitro differentiation potential of mesenchymal stem cells. Transfus Med Hemother 35: 228-238, 2008

3. Toupadakis CA, Granick JL, Sagy M, et al: Mobilization of endogenous stem cell populations enhances fracture healing in a murine femoral fracture model. Cytotherapy 15: 1136-1147, 2013.

4. Corallini F, Secchiero P, Beltrami AP, et al: TNF-alpha modulates the migratory response of mesenchymal stem cells to TRAIL. Cell Mol Life Sci 67: 1307-1314, 2010.

5. Wong HL, Siu WS, Shum WT, et al: Application of Chinese herbal medicines to revitalize adult stem cells for tissue regeneration. Chin J Integr Med 18: 903-908, 2012.

6. Krampera M,Franchini M,Pizzolo G and Aprili G: Mesenchymal stem cells: from biology to clinical use. Blood Transfus 5: 120-129, 2007.

7. Zhang HT, Liu ZL, Yao XQ, Yang ZJ and Xu RX: Neural differentiation ability of mesenchymal stromal cells from bone marrow and adipose tissue: a comparative study. Cytotherapy 14: 1203-1214, 2012. 
8. Hass R, Kasper C, Böhm S and Jacobs R: Different populations and sources of human mesenchymal stem cells (MSC) A comparison of adult and neonatal tissue-derived MSC. Cell Commun Signal 9: 12, 2011.

9. Yoshimura H, Muneta T, Nimura A, et al: Comparison of rat mesenchymal stem cells derived from bone marrow, synovium, periosteum, adipose tissue, and muscle. Cell Tissue Res 327: 449-462, 2007.

10. Fan J, Varshney RR, Ren L, Cai D and Wang DA: Synovium-derived mesenchymal stem cells: a new cell source for musculoskeletal regeneration. Tissue Eng Part B Rev 15: 75-86, 2009.

11. Matsubara T, Suardita K, Ishii M, et al: Alveolar bone marrow as a cell source for regenerative medicine: differences between alveolar and iliac bone marrow stromal cells. J Bone Miner Res 20: 399-409, 2005.

12. Rui YF, Lui PP, Ni M, et al: Mechanical loading increased BMP-2 expression which promoted osteogenic differentiation of tendon-derived stem cells. J Orthop Res 29: 390-396, 2011.

13. Ikegame $\mathrm{Y}$, Yamashita $\mathrm{K}$, Hayashi S, et al: Comparison of mesenchymal stem cells from adipose tissue and bone marrow for ischemic stroke therapy. Cytotherapy 13: 675-685, 2011.

14. Vidal MA, Walker NJ, Napoli E and Borjesson DL: Evaluation of senescence in mesenchymal stem cells isolated from equine bone marrow, adipose tissue, and umbilical cord tissue. Stem Cells Dev 21: 273-283, 2012.

15. Taléns-Visconti R, Bonora A, Jover R, et al: Hepatogenic differentiation of human mesenchymal stem cells from adipose tissue in comparison with bone marrow mesenchymal stem cells. World J Gastroenterol 12: 5834-5845, 2006.

16. Tao H, Yu MC, Yang HY, et al: Correlations between fasciology and yin yang doctrine. J Acupunct Meridian Stud 4: 141-146, 2011.

17. Wang J, Wang CL, Shen BL, Yang LL and Yuan L: Explanation of essence and substance basis of channels and collaterals with fasciology. Zhongguo Zhen Jiu 27: 583-585, 2007 (In Chinese).

18. Li G, Zheng B, Meszaros LB, et al: Identification and characterization of chondrogenic progenitor cells in the fascia of postnatal skeletal muscle. J Mol Cell Biol 3: 369-377, 2011.

19. Ko CH, Siu WS, Wong HL, et al: Pro-bone and antifat effects of green tea and its polyphenol, epigallocatechin, in rat mesenchymal stem cells in vitro. J Agric Food Chem 59: 9870-9876, 2011.

20. Stecco C, Tiengo C, Stecco A, et al: Fascia redefined: anatomical features and technical relevance in fascial flap surgery. Surg Radiol Anat 35: 369-376, 2013.

21. Schleip R, Jäger H and Klingler W: What is 'fascia'? A review of different nomenclatures. J Bodyw Mov Ther 16: 496-502, 2012.

22. Schäffler A and Büchler C: Concise review: adipose tissue-derived stromal cells--basic and clinical implications for novel cell-based therapies. Stem Cells 25: 818-827, 2007.
23. De Ugarte DA, Alfonso Z, Zuk PA, et al: Differential expression of stem cell mobilization-associated molecules on multi-lineage cells from adipose tissue and bone marrow. Immunol Lett 89 : 267-270, 2003.

24. Zuk PA,Zhu M, Ashjian P, et al: Human adipose tissue is a source of multipotent stem cells. Mol Biol Cell 13: 4279-4295, 2002.

25. Steinert AF, Rackwitz L, Gilbert F, Nöth U and Tuan RS: Concise review: the clinical application of mesenchymal stem cells for musculoskeletal regeneration: current status and perspectives. Stem Cells Transl Med 1: 237-247, 2012.

26. Kern S, Eichler H, Stoeve J, Klüter H and Bieback K: Comparative analysis of mesenchymal stem cells from bone marrow, umbilical cord blood, or adipose tissue. Stem Cells 24: 1294-1301, 2006.

27. Peng L, Jia Z, Yin X, et al: Comparative analysis of mesenchymal stem cells from bone marrow, cartilage, and adipose tissue. Stem Cells Dev 17: 761-773, 2008.

28. Potier E, Ferreira E, Meunier A, et al: Prolonged hypoxia concomitant with serum deprivation induces massive human mesenchymal stem cell death. Tissue Eng 13: 1325-1331, 2007.

29. Greco SJ, Liu K and Rameshwar P: Functional similarities among genes regulated by OCT4 in human mesenchymal and embryonic stem cells. Stem Cells 25: 3143-3154, 2007.

30. Li Z, Tian X, Yuan Y, et al: Effect of cell culture using chitosan membranes on stemness marker genes in mesenchymal stem cells. Mol Med Rep 7: 1945-1949, 2013.

31. Rasini V, Dominici M, Kluba T, et al: Mesenchymal stromal/stem cells markers in the human bone marrow. Cytotherapy 15: 292-306, 2013

32. Thomson M, Liu SJ, Zou LN, et al: Pluripotency factors in embryonic stem cells regulate differentiation into germ layers. Cell 145: 875-889, 2011

33. Saulnier N, Puglisi MA, Lattanzi W, et al: Gene profiling of bone marrow- and adipose tissue-derived stromal cells: a key role of Kruppel-like factor 4 in cell fate regulation. Cytotherapy 13: 329-340, 2011.

34. Wu X, Ren J and Li J: Fibrin glue as the cell-delivery vehicle for mesenchymal stromal cells in regenerative medicine. Cytotherapy 14: 555-562, 2012.

35. De Ugarte DA, Morizono K, Elbarbary A, et al: Comparison of multi-lineage cells from human adipose tissue and bone marrow. Cells Tissues Organs 174: 101-109, 2003.

36. Zhang W, Zhang X, Wang S, et al: Comparison of the use of adipose tissue-derived and bone marrow-derived stem cells for rapid bone regeneration. J Dent Res 92: 1136-1141, 2013.

37. Tan Q, Lui PP, Rui YF and Wong YM: Comparison of potentials of stem cells isolated from tendon and bone marrow for musculoskeletal tissue engineering. Tissue Eng Part A 18: 840-851, 2012.

38. Wegmeyer H, Bröske AM, Leddin M, et al: Mesenchymal stromal cell characteristics vary depending on their origin. Stem Cells Dev 22: 2606-2618, 2013. 